\title{
Gestión sobre investigación operativa en toxicología ocupacional mediante modelo dinámico de estrategia planeada
}

Management on operational research in occupational toxicology using the dynamic model of planned strategy

Recibido: julio 11 de 2016 | Revisado: agosto 10 de 2016 | Aceptado: setiembre 15 de 2016

\author{
George Argota Pérez \\ Alembert Coasaca NúñEZ ${ }^{2}$
}

\begin{abstract}
Resumen
El objetivo de esta investigación fue describir la gestión sobre investigación operativa en toxicología ocupacional mediante un modelo dinámico de estrategia planeada. Para ello, se analizó el desempeño del Laboratorio de Toxicología Ocupacional, perteneciente al Centro de Toxicología y Biomedicina en Santiago de Cuba, Cuba. Se aplicó como método cuantitativo de investigación el teórico sistemático, así como el empírico por medición. La técnica cuantitativa de análisis se basó en la observación estructurada. La gestión operacional del modelo dinámico de estrategia planeada, difirió de cualquier otro existente en Cuba, ya que la manera de competir, entre los centros toxicológicos por los sectores de la sociedad, es muy diferente dada la misión que desempeñan, el desarrollo de sus recursos materiales, financieros y humanos, así como las capacidades de organización que presentan. El carácter de operatividad desarrollado mediante las cinco etapas, incluyen pensamiento estratégico, análisis estratégico, formulación estratégica, programación estratégica e implantación y control estratégico. Las etapas correspondieron a los servicios preventivos que se realizan con base en asesorías de evaluación de riesgo toxicológico ocupacional, las que permiten determinar y aplicar, de forma científica, nuevas políticas de acción. Se concluyó que el modelo dinámico, descrito sobre la estrategia planeada a seguir para los servicios científicotécnicos e investigaciones biomédicas en toxicología ocupacional, permitió visualizar, conjuntamente con las funciones administrativas y directivas las diferentes posibilidades de implementar y coordinar diagnósticos preventivos y de pesquisas activas, con base en asesorías de evaluación de riesgo toxicológico ocupacional, que posibilitó determinar y aplicar científicamente nuevas políticas de acción.
\end{abstract}

Palabras claves: estrategia planeada, gestión operacional, modelos de gestión dinámicos, toxicología ocupacional, Santiago de Cuba-Cuba
Abstract
The objective of this research was to describe the management on operational research in occupational toxicology using a dynamic model of planned strategy. In order to do this, we analyzed the 
performance of the Laboratory of Occupational Toxicology, belonging to the Center for Toxicology and Biomedicine in Santiago de Cuba, Cuba. The quantitative methods of research applied were the systematic-theoretical as well as the empirical by measurement. The quantitative technique of analysis was based on structured observation. The operational management of the dynamic model of planned strategy differed from any other existing in Cuba, since the way to compete between the toxicological centers by sectors of society is very different given the mission they carry out, the development of their material, financial and human resources, as well as the organizational capacities they possess. The operational character can be separated into five stages, which include strategic thinking, strategic analysis, strategic formulation, strategic programming and implementation and strategic control. The stages correspond to the preventive services that are carried out based on assessments of occupational toxicological risk, which will allow us to determine and apply, scientifically, new policies of action. It was concluded that the dynamic model, described on the planned strategy to be followed for scientific-technical services and biomedical investigations in occupational toxicology, allowed us to visualize, together with the administrative and managerial functions, the different possibilities of implementing and coordinating preventive diagnoses and active research , based on assessments of occupational toxicological risk, which made it possible to scientifically determine and apply new action policies.

Palabras clave: planned strategy, operational management, dynamic management models, occupational toxicology, Santiago de Cuba-Cuba

\section{Introducción}

Resulta relevante mencionar que diversas tareas desempeñadas por los trabajadores pueden tener consecuencias desagradables, nocivas e incluso desastrosas para ellos. La gran mayoría de estas consecuencias pueden prevenirse, pero desafortunadamente, los conocimientos que permiten tales acciones, distan de ser aplicados de manera universal, ya que solo una pequeña proporción de los trabajadores del mundo en desarrollo está cubierto por programas sociales y estos no incluyen, en la práctica, la toxicología ocupacional. Asimismo, es necesario indicar que no es lo mismo salud en el trabajo, medicina del trabajo y toxicología ocupacional, ya que ninguna de las especialidades son equivalentes. La salud, en el trabajo, se refiere a una perspectiva amplia que incluye diferentes disciplinas, donde la medicina del trabajo es uno de los elementos (OMS, 1995).

Gastañega (2012), en su trabajo denominado "Salud ocupacional: historia y retos del futuro" interpreta, de forma general, que si bien es cierto que el crecimiento económico sostenido tiene su base en la población laboral, ello de igual forma debe considerar que podría generarse determinadas inseguridades, por cuanto la salud de los trabajadores tiene por objeto el control de los riesgos ocupacionales, protección y promoción de la salud, así como la huma- 
nización del trabajo aportando, por una parte, conocimientos científicos; y por otra parte, orientación a las autoridades que han de adoptar decisiones sobre la legislación sanitaria y su aplicación.

La Organización Mundial de la Salud (2010) indica que la salud de los trabajadores tiene por objeto el control de los riesgos ocupacionales, la protección y promoción de la salud, así como la humanización del trabajo, que aporta por una parte, conocimientos científicos; y por la otra, orientación a las autoridades que han de adoptar decisiones sobre la legislación sanitaria y su aplicación, por cuanto deberán implementarse investigaciones operativas que finalmente posibiliten garantizar la salud de los trabajadores en función a mejores decisiones por parte de las direcciones y/o administraciones.

La investigación operativa (IO) en salud, disciplina poco desarrollada todavía, tiene como objetivo proporcionar información útil a las personas que tienen el poder de tomar decisiones para mejorar las políticas en salud a nivel local, regional o nacional. La IO tiene como particularidad que las preguntas de investigación surjan de problemas detectados en la implementación de proyectos de salud en el terreno; y que las respuestas a estas preguntas pretenden solucionar estas dificultades (Hernández, 2013).

Para entender qué es la investigación operativa, es necesario situar la misma dentro de un esquema global. Existen varios modelos e intentos de clasificar los distintos tipos de investigación científica. Estos incluyen desde el denominado "modelo de ocho fases" hasta el "modelo de dos fases", pasando por el "modelo de cinco fases" (Sussman, Valente, Rohrbach, Skara \& Pentz, 2006). Tanto el modelo de ocho fases como el de cinco o el de dos, sitúan a las investigaciones científicas en una línea de tiempo ideal que va desde la investigación básica hasta la aplica$\mathrm{da}$, pasando por todas las etapas necesarias hasta llegar a su implementación óptima en diversos escenarios reales.

Por su sencillez y practicidad, se empleó el modelo de dos fases, en que los estudios se dividen en tipo I y tipo II. Los estudios de tipo I incluyen, a su vez, la investigación básica y la investigación de eficacia. La investigación básica se desarrolla en el laboratorio y tiene como finalidad proporcionar explicaciones teóricas para fenómenos naturales de interés. La investigación de eficacia evalúa el impacto inicial de la intervención estudiada bajo condiciones controladas, que pueden ser, en el laboratorio u otro tipo de condiciones limitadas (Rabin, 2008). Así, los resultados de las investigaciones de tipo I no pueden extrapolarse a la rutina diaria de implementación en condiciones "reales". Los estudios de tipo II incluyen la denominada investigación de efectividad, que pretende demostrar la eficacia de una intervención en condiciones "reales"; la investigación de diseminación, que estudia cuál es la mejor estrategia para extender un tipo de intervención en la población meta de forma que esta la adopte y la utilice; y finalmente, la investigación operativa (IO), que estudia y busca la adaptación de las intervenciones a un contexto local específico para que funcionen de forma óptima.

Rubio et al. (2010) mencionan que en la investigación operativa es importante destacar que también se conoce como investigación de operaciones, investigación de ejecución (IE) (implementation research en inglés) e incluso un subtipo de la investigación de transferencia (del inglés, translational research).

Según Rojas (1998), la toxicología ocupacional es la ciencia que estudia los efectos adversos a la salud producidos por agentes químicos utilizados y a los que están expuestos los trabajadores como consecuencia de su manipulación, elaboración y uso. A la par menciona, que estudia los mecanismos 
de acción de dichos tóxicos y las formas de prevenir y controlarlos, siendo su objetivo principal la prevención de los daños y solo puede alcanzarse, si las condiciones de exposición a estos agentes peligrosos y las prácticas de trabajo se definen de tal forma, que no produzcan un riesgo inaceptable a la salud humana.

De acuerdo a Niño (2000), la misión de estrategia sobre la prevención en toxicología ocupacional (EPTO), es tomada a menudo como una misión imposible. Las dificultades conceptuales y de aplicación, han hecho parecer, en innumerables ocasiones a la prevención, un propósito entre titánico y trivial de manera que se necesita, pasar desde una cultura de las condolencias y las lamentaciones en el trabajo, a una cultura de la prevención efectiva laboral; y esto es un proceso complejo que lleva mucho tiempo. Progresar desde una prevención de las evidencias de los peligros a una prevención de los invisibles riesgos, requiere conjuntamente de la voluntad y determinación, un desarrollo técnico y conceptual, que se extraña, en muchos de los planteamientos actuales para que la prevención de los accidentes y las enfermedades laborables sean tanto creíbles como viables.

El estudio de la EPTO debe hacer referencia a dos aspectos fundamentales que si bien, en la teoría, son claramente diferenciables, en la práctica son difíciles de separar. Por una parte, se puede analizar el contenido de la estrategia y por la otra, el proceso que se sigue en la organización toxicológica para la formación de la propia estrategia. Esto ha conducido, según Rajagopalan (1997), a la creación de dos escuelas de pensamientos diferentes: la escuela del contenido, centrada en los antecedentes y consecuencias del cambio estratégico, donde lo importante es poder definir en qué consiste la estrategia y comprender cuáles son los elementos que la componen para conseguir alcanzar o bien mantener una ventaja competitiva; y la escuela del proceso, la cual está interesada en el papel que juegan los directivos en el proceso de cambio estratégico.

Schendel (1992) justifica la necesidad de mantener tal dicotomía en el pensamiento de ambas escuelas, ya que en la del contenido, es conveniente hacer referencia a cómo se va configurando la estrategia realmente en la organización y por otro lado, se toma en consideración el papel gestor de los directivos, que obliga a centrar la atención a todos los participantes en los aspectos esenciales para el futuro de la misma. Según Cejas (2006), Castellanos \& Cruz (2014) y Quero (2008), entre los factores más importantes que afectan el éxito en la implantación de la estrategia, todas las organizaciones citan a las personas como un recurso de alta valoración por competencia, manifestándose de esta forma, una gran preocupación por las represiones que sobre el recurso humano tiene la implantación de una nueva estrategia y paralelamente, la necesidad de contar con él para poder implantarla.

En el campo de la toxicología ocupacional, son varios los modelos, patrones y matrices, entre otras herramientas estratégicas investigativas, que se utilizan para introducir nuevos cambios de acción que permitan generar, operativamente, mejores valores de impacto en materia de preservar la salud laboral (Tamayo \& Argota, 2012). En tal sentido, puede considerarse que la salud de los trabajadores es estimada por los organismos internacionales como tema prioritario, ya que el costo social por su deterioro es un obstáculo para el desarrollo sostenible de cualquier país, por tal motivo la medicina del trabajo, dedica al estudio del proceso salud-enfermedad, una contribución que promueva la salud sobre los riesgos que entraña cualquier actividad para lo cual, requiere especialistas con actitud crítica y reflexiva que privilegien el conocimiento sobre estos valores, en pos de una mejor calidad técnica y humana que evite desvirtuar su quehacer. 
Finalmente, las EPTO seguidas por las organizaciones, deben ser más que simples sistemas formalizados de planificación y acumulación de acciones aisladas y oportunistas, sino que deberán basarse en lo más habitual que es la integración de diferentes modelos estratégicos.

El objetivo de la presente investigación fue la gestión operacional en toxicología ocupacional mediante un modelo dinámico de estrategia planeada.

\section{Método \\ Objeto de investigación y periodo de estudio}

Se analizaron las características funcionales de desempeño correspondientes al Laboratorio de Toxicología Ocupacional del Centro de Toxicología y Biomedicina (TOXIMED) de Santiago de Cuba-Cuba, durante los años 2008 hasta 2011.

\section{Población y muestra}

Se estudiaron las características correspondientes a los servicios, servicios científico-técnicos, así como las investigaciones biomédicas en toxicología ocupacional (Tabla 1) y seleccionaron mediante un muestreo no probabilístico por conveniencia, aquellos que presentaron una marca agua con relación a su visibilidad.

Tabla 1

Modalidades y caracteristicas de la toxicología ocupacional

\begin{tabular}{|c|c|}
\hline MODALIDAD & CARACTERÍSTICAS \\
\hline Servicios & $\begin{array}{l}\text { a. Servicios de consultoría toxicológica ocupacional } \\
\text { - Información recogida en fichas de consultas y manejo de sistemas } \\
\text { de gestión de bases de datos (se establece comunicación telefónica). } \\
\text { b. Servicios de asesorías de evaluación de riesgo toxicológico ocupacional } \\
\text { basadas en fichas técnicas } \\
\text { - Realizar pruebas médicas o exámenes físicos a los trabajadores. } \\
\text { - Revisar los registros de lesiones y enfermedades, los datos sobre } \\
\text { exposiciones de los trabajadores a los agentes peligrosos, las pruebas } \\
\text { médicas y las historias ocupacionales. }\end{array}$ \\
\hline $\begin{array}{c}\text { Servicios } \\
\text { científico-técnicos } \\
\end{array}$ & $\begin{array}{l}\text { Se incluyen las actividades de la modalidad del servicio además de la } \\
\text { orientación con realización de perfiles toxicológicos o sin ella. }\end{array}$ \\
\hline $\begin{array}{l}\text { Investigaciones } \\
\text { biomédicas }\end{array}$ & $\begin{array}{l}\text { Se incluyen las actividades de las modalidades del servicio como de } \\
\text { servicios científico-técnicos, además, de la determinación y evaluación } \\
\text { de biomarcadores de daños (biológicos, físicos y químicos); evidencia de } \\
\text { cualquier evento centinela en toxicología ocupacional; aprobación del } \\
\text { consentimiento informado. }\end{array}$ \\
\hline
\end{tabular}

\section{Análisis de las variables}

Teniendo como base la gestión del conocimiento para el desarrollo del modelo dinámico de estrategia planeada, en toxicología ocupacional, (Figura 1), se analizó entonces el comportamiento que caracterizó a las siguientes etapas:

- Pensamiento estratégico (I), - análisis estratégico (II), - formulación estratégica (III),
- Programación estratégica (IV), - implantación y control estratégico (V).

La Figura 1 representa el diagrama sobre el cual se basa el modelo dinámico de estrategia planeada, creado para los estudios en toxicología ocupacional del Centro de Toxicología y Biomedicina de Santiago de Cuba. 


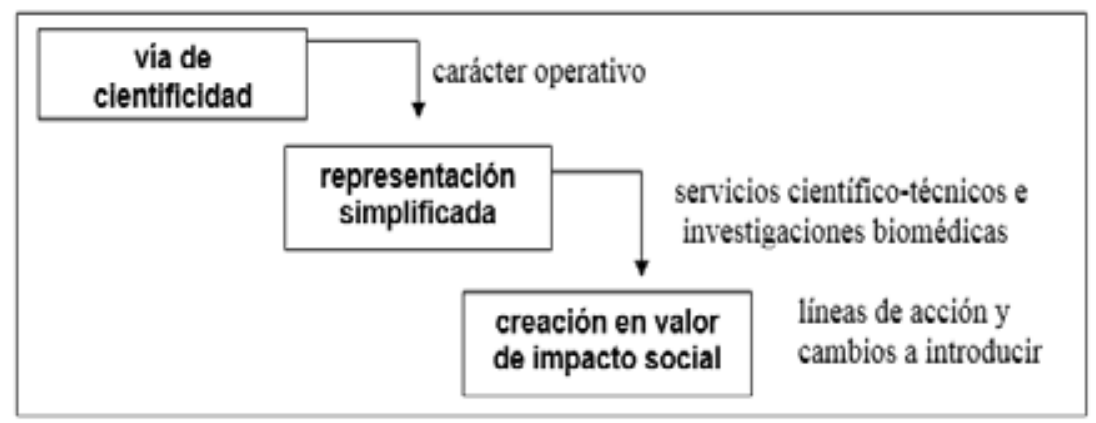

Figura 1. Diagrama base de la gestión del conocimiento para el desarrollo del modelo dinámico de estrategia planeada toxicológico-ocupacional

\section{Resultados}

La estrategia propuesta para los estudios, en el campo de la toxicología ocupacional, estuvo caracterizada por cinco etapas en las que se definen el pensamiento estratégico, análisis estratégico, formulación estratégica, programación estratégica e implantación y control estratégico.
El pensamiento estratégico hace referencia a la conveniencia o no de cambiar lo que se está haciendo en el presente para tener un futuro mejor. La respuesta viene dada como se puede observar en la Figura 2, por la definición de la misión, capacidades organizativas, valores humanos, momentos de la estrategia, viabilidad de acción y visión en el campo de la actividad.

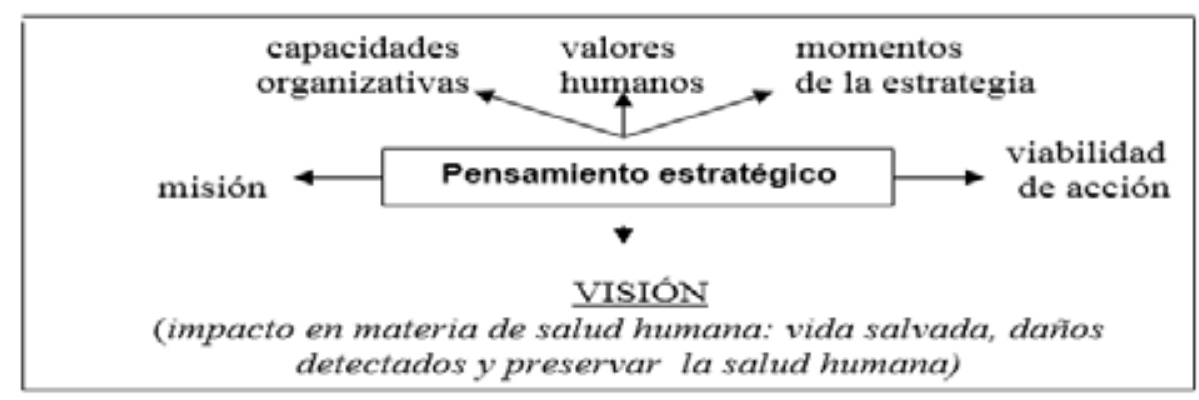

Figura 2. Características del pensamiento estratégico en toxicología ocupacional

En el análisis estratégico, se hace un diagnóstico de la situación de la organización frente a las variables que caracterizan el reto estratégico. En la Figura 3, se muestra que dicha etapa está caracterizada por dos aspectos: uno interno y otro externo, en los cuales aparecen algunas de sus identidades.

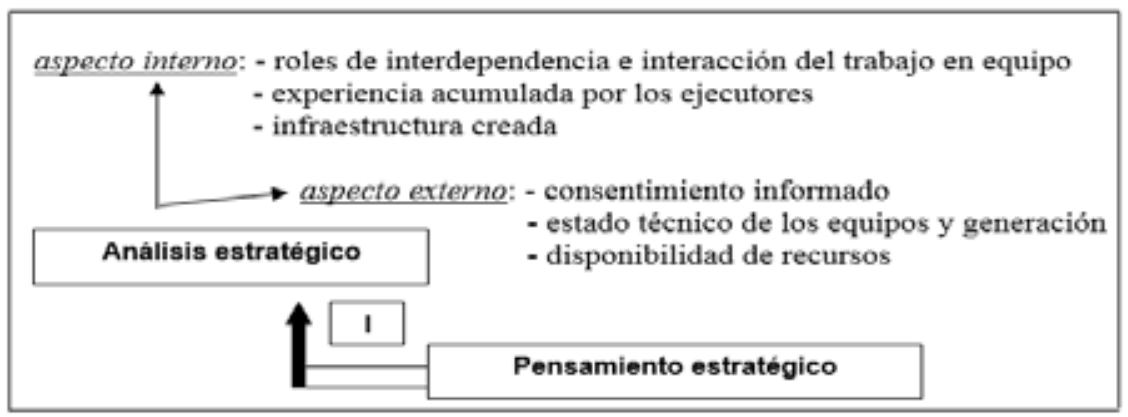

Figura 3. Características del análisis estratégico en toxicología ocupacional 
La etapa formulación estratégica (Figura 4) se basa en la identificación y evaluación de diferentes alternativas estratégicas. Se culmina con la selección de una estrategia en específico. En esta etapa, aparecen algunas herramientas que pueden ser implementadas para el desarrollo del trabajo toxicológico ocupacional.

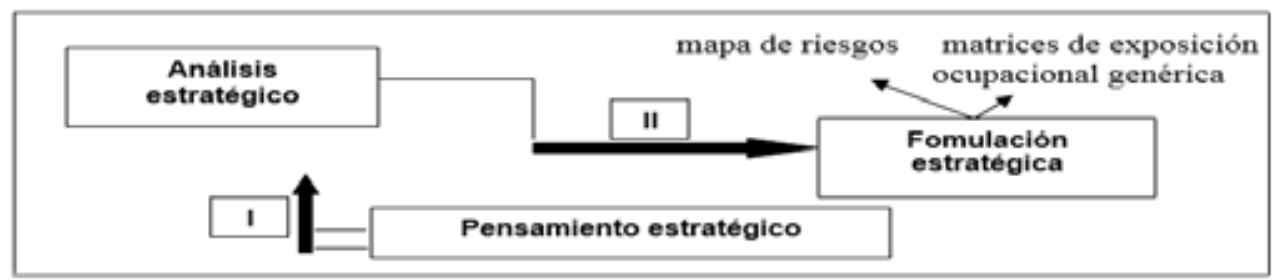

Figura 4. Características de la formulación estratégica en toxicología ocupacional

En la etapa programación estratégica (Figura 5), se concreta la estrategia seleccionada de la etapa anterior evaluando sus implica- ciones, apareciendo, además, en esta etapa, algunos planes que pueden concebirse.

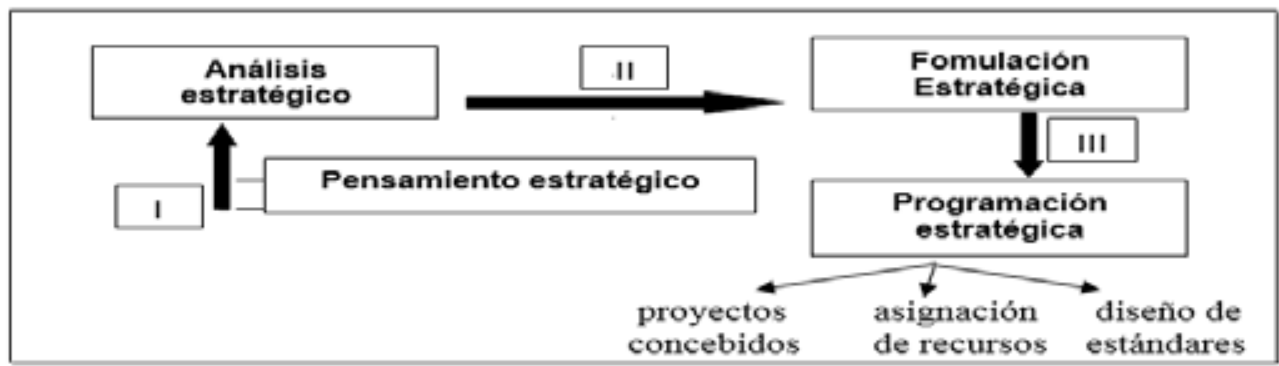

Figura 5. Características de la programación estratégica en toxicología ocupacional

En la etapa implantación y control estratégico (Figura 6), se hace referencia a las actividades que realmente se están realizando en el servicio de investigaciones y las múltiples decisiones que se toman a lo largo del ejercicio institucional con relación al trabajo toxicológico ocupacional. Se menciona que los servicios científico - técnicos e investigaciones biomédicas son realizados con base en asesorías de evaluación de riesgos-toxicológico ocupacional (AER-TO), consulta diagnosticada al trabajador y gestión de entrega de informes.

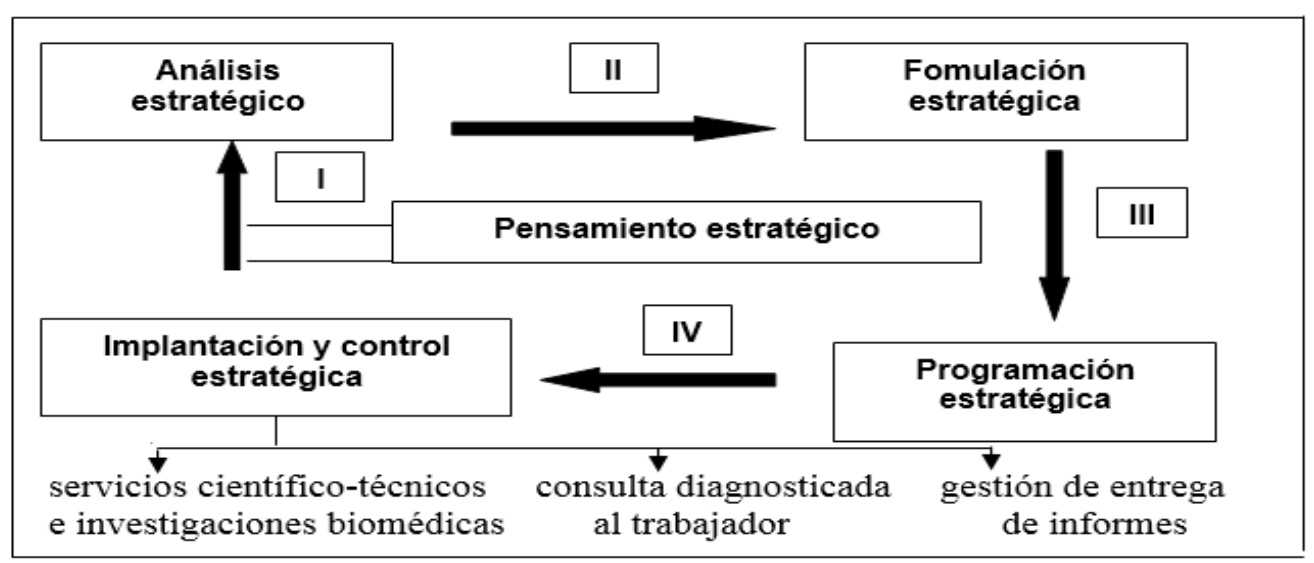

Figura 6. Características de la implantación y control estratégico en toxicología ocupacional 
En la Figura 7 se muestran otras dos variantes estratégicas que caracterizan todo el trabajo toxicológico ocupacional, ya que se ha considerado, por una parte, la misión institucional, donde esta disciplina toxicológica es la única que se realiza diferente con rela- ción al resto de los centros toxicológicos y otras instituciones existentes en el país. Asimismo, se presentan las tres variantes estratégicas que conforman el modelo dinámico de estrategia planeada según la gestión del conocimiento aplicado.

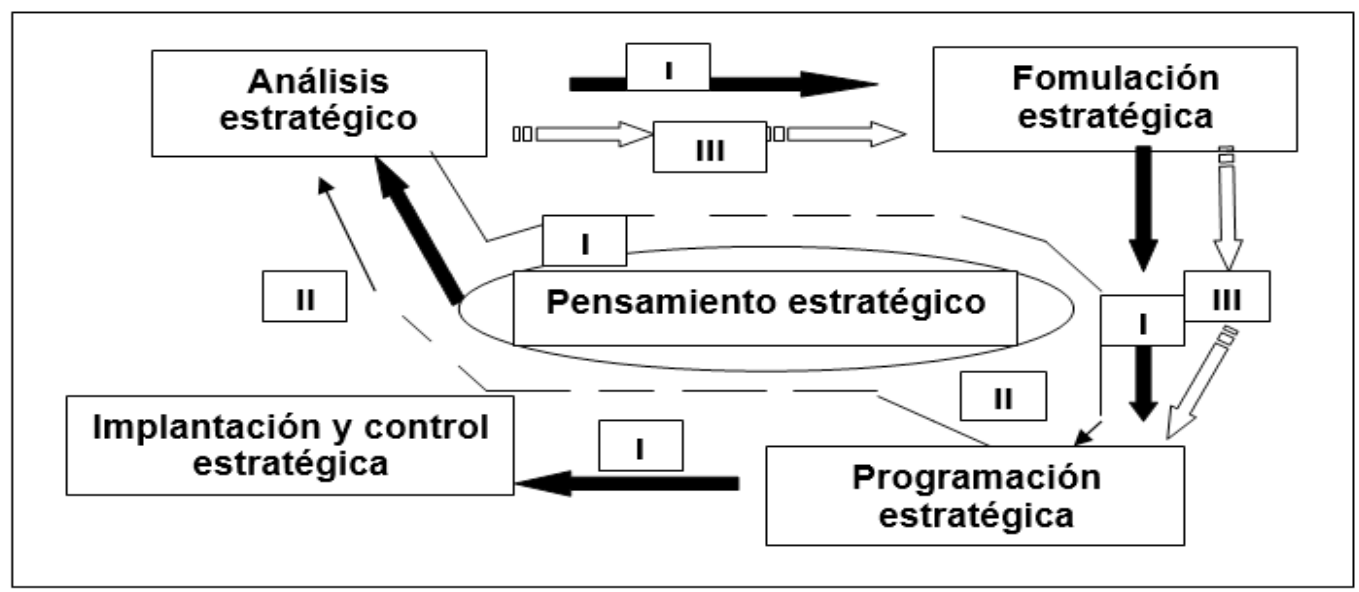

Figura 7. Modelo dinámico de estrategia planeada según la gestión del conocimiento para la toxicología ocupacional

\section{Discusión}

El desarrollo de las actividades en las organizaciones toxicológicas requiere utilizar métodos que permitan la adopción racional de las decisiones, disponiendo de medios para coordinar las actividades, normas para comunicar las informaciones y procedimientos de éxitos en las evaluaciones de control organizacional, en cuanto al alcance de los objetivos fijados, así como adoptar las decisiones indispensables, a fin de rectificar la marcha de los procesos toxicológicos cuando los objetivos pudieran verse afectados. Toda institución constituye en sí, un sistema integrado por numerosos subsistemas dotados de elementos y relaciones, que es la base de su complejidad. Algunos de los mecanismos directivos en las investigaciones son eficaces; otros resultan indudablemente endebles, pero a pesar de los buenos o malos resultados con que cumplen su cometido, son elementos indispensables de excepcional importancia.

Puede entenderse que, en la actualidad, la atención mundial sobre la salud laboral, transita entre nuevos paradigmas que buscan mejoras en razones económicas, fidelización de los sectores productivos, así como posicionamientos en torno al reconocimiento social bajo funciones directivas de las organizaciones, siendo los indicadores, anteriormente mencionados, razones de alto significado y es lo que puede reconocerse como Gestión del Conocimiento en Investigación Operativa sobre el campo de la atención a la salud pública.

La gestión del conocimiento en toxicología ocupacional tiene que facilitar el aumento y desarrollo de la manera de pensar para la mejora simultánea de productividad y competitividad. El cambio de estrategia afecta al comportamiento de las personas, introduciendo cambios en su manera de operar $y$, en muchos casos, modificando las estructuras organizativas institucionales. Tanto el contenido como el proceso estratégico en su conjunto, constituyen elementos de vital importancia para el control de los trabajos toxicológicos porque en sus distintas etapas, obliga a centrar la atención de todos los par- 
ticipantes en los aspectos esenciales para el futuro de la organización.

El modelo dinámico de estrategia obtenido (Figura 7) pretende adoptar una estructura circular mucho más completa que cualquier modelo lineal o secuencial en la que no existe ni un claro principio ni final, así como tampoco está definido, de forma unívoca, el camino a seguir. Las flechas que se indican, no implican que haya que seguir un orden secuencial, ni que tengan que cumplirse necesariamente todas ellas. Sin embargo, las secuencias del modelo al ser una simplificación de la realidad, indican un orden lógico de preferencia, aunque en el funcionamiento de cualquier organización toxicológica se pueden suponer determinadas etapas y realizarse varias de ellas de forma simultánea.

El modelo presentado representa las distintas posibilidades de ofrecer con garantía, mejores y eficaces servicios científico-técnicos e investigaciones para la toxicología ocupacional y en tal sentido, se ha concebido las tres variantes dinámicas a seguir de acuerdo con las posibilidades reales del trabajo toxicológico. Las diferentes etapas representadas tratan de relacionarse como procesos integrados coincidiendo con lo planteado por Chakravarthy y Doz (1992), quienes mencionan que los procesos estratégicos deben valorarse como estrategias efectivas validadas de forma racional y consciente, ya sea mediante sistemas de planificación formalizados o mediante la acumulación de acciones.

Por otra parte, el modelo ha tenido en cuenta lo planteado por Hax \& Majluf (1996), indicando que la estrategia emerge en la organización como consecuencia de múltiples acciones, siendo algunas de ellas planteadas de forma explícita y que es consecuencia fundamentalmente de los planes generados por el sistema formal toxicológico. La estrategia seguida es planeada, ya que el Centro de Toxicología y Biomedicina, es el único de su tipo en el país que por misión ofrece servicios científico-técnicos e investigaciones toxicológica-ocupacionales; y esto representa su carácter de planificación, de manera que las tres variantes dinámicas consideran y justifican lo que se implementa en este campo.

La variante uno del modelo, representa un ciclo completo caracterizado por cinco etapas. Durante el pensamiento estratégico, es concebido el trabajo toxicológico ocupacional con base en asesorías de evaluación de riesgos. Las AER deben ser etapas preliminares ante cualquier estudio de evaluación de riesgos, ya que las mismas ofrecen informaciones tanto cualitativas como cuantitativas de daños asociados a eventos, acciones, sustancias y procesos que provocan no solo efectos adversos a la salud humana, sino también a los ecosistemas (Albert, 1997).

A partir de estas AER se ha tenido como visión proyectada, identificar en materia de impacto problemas detectados como vida salvada. En esta etapa además, al concentrarse los esfuerzos para tener un futuro mejor, se definen nuevas formas de competencias caracterizadas por un entorno en el que los ciclos de vida de los servicios que las organizaciones toxicológicas ofrecen $\mathrm{o}$ incluyen, pueden reducirse siendo más imprescindibles, que se corresponden con los estudios de flexibilidad estratégica realizados por Sánchez (1995), quien plantea que esta capacidad, en algunas organizaciones, responden más rápidamente a los cambios en las oportunidades por ocupar sectores de la población no cubiertos, ofrecer servicios más baratos y en determinadas circunstancias que sean bien diferentes.

En el análisis estratégico, se han señalado dos aspectos: uno interno que se centra en el diagnóstico y valoración de los recursos y capacidades de la organización que se han definido en el pensamiento estratégico, mientras que el externo se concreta en el seguimiento periódico de las variables que influyen en el 
entorno general del centro y en su campo de actividad, siendo muy importante señalar que, en algunas instituciones, esta etapa es altamente formalizada y respondida por un departamento de staff que utiliza métodos y técnicas muy sofisticadas, mientras que en otras, es de una forma mucho más simple e informal, que se realiza en función de las necesidades que vayan surgiendo. Las variables identificadas en esta etapa, constantemente van cambiando debido, fundamentalmente, al progreso tecnológico experimentado en las tecnologías de las información y en la metodología del diseño de evaluaciones que caracterizan la actividad toxicológica ocupacional, coincidiendo con lo planteado por Mallar (2010), sobre la gestión de los recursos tanto económicos, tecnológicos, organizativos como los humanos.

Con relación a la formulación estratégica, hay que señalar que, en algunos casos, las herramientas son desarrolladas, en fases posteriores al proceso estratégico, mediante una programación detallada de los mismos para su puesta en marcha, mientras que en otros casos se incorporan simultáneamente a la acción estratégica. En esta etapa, se estudian los modelos, herramientas, patrones y matrices como las de exposición ocupacional genérica ocupacional las cuales posibilitan a partir de su consistencia y validez, estimar el riesgo a la salud de los trabajadores (Haro, Celis, López, Sánchez \& Juárez, 2007).

En la etapa de programación estratégica, se concibe la elaboración de los distintos planes toxicológicos ocupacionales que, en cierta medida, culminan presentándose en forma corporativa y son establecidos y evaluados además, programas de acción en el ámbito funcional y unidades de negocio, así como en la asignación de recursos y diseño de estándares para el establecimiento de los sistemas de control y las implicaciones de asignación de inversiones entre los distintos proyectos.
La etapa implantación y control estratégico implica una forma determinada de utilización de los recursos y comprenden los distintos sistemas establecidos para accionar, de manera gestora, el funcionamiento del trabajo toxicológico-ocupacional.

En este período, se han establecido fichas técnicas para las asesorías de evaluación de riesgo toxicológico ocupacional donde se podrá estar en mejores condiciones de conocer las características de la exposición, establecer el monitoreo biológico adecuado, el tipo de biomarcador a seleccionar y el tiempo de la toma de la muestra con respecto a la exposición e interpretación de los resultados, permitir criterios de peligrosidad de las sustancias y de riesgos, así como las características de los efectos tóxicos como las interacciones de los agentes químicos, todos los cuales se corresponden con lo planteado por Albert (1997) para las evaluaciones de riesgo toxicológico.

La variante dinámica dos, generada, reafirma que la estrategia es planeada siendo el resultado de un sistema de planificación caracterizado por la "ausencia de cambio", donde esta variante tiende, a que el trabajo toxicológico-ocupacional permanezca constante en el tiempo, en que la etapa formulación estratégica desaparece (Figura 7) y solamente se actualizan los planes de forma periódica que, en cierto modo, van perdiendo su carácter estratégico y se convierte en operacional con lo que la estrategia es el reflejo de esas operaciones.

Es importante mencionar que los sistemas formales de planificación estratégica, han recibido fuertes críticas, en los últimos tiempos, por su escasa operatividad. Mintzberg (1994) ha puesto de manifiesto, en distintas ocasiones, que los sistemas de planificación no producen estrategias, sino planes y una cosa son los planes y otra el comportamiento real de la organización. 
La variante dinámica tres caracteriza el trabajo toxicológico-ocupacional, realizado en entornos "estables" y aunque se ha considerado que el mismo es diferente al realizado por otras instituciones existentes, en el país, como por ejemplo, el Instituto Nacional para la Salud de los Trabajadores (INSAT), en cierta medida se considera importante hacer frente a determinados cambios los cuales, necesariamente, deberán ejecutarse en la práctica sin que ellos constituyan agravar el sistema de planificación existente.

Como ha planteado Hamel (1998a), la solución a la nueva situación no está en repetir los procesos estratégicos tradicionales, sino que de alguna manera tiene que reinventarse la práctica de la estrategia siendo importante, que no es centrarse en la estrategia en sí, sino en crear las precondiciones necesarias que hagan posible el surgimiento de la innovación estratégica. Como puede observarse, el énfasis no está en el diseño del proceso ni en la secuencia de las etapas, sino en las condiciones previas o las actitudes en las que se van a desarrollar el trabajo toxicológico-ocupacional. Sin embargo, la introducción de todas estas novedades en la estrategia de esta disciplina, implica de alguna forma que se establezcan algunos mecanismos formales que permitan nuevos servicios de manera coordinada.

Coincidiendo con Van de Ven (1992), quien menciona que los procesos, en cualquier modelo estratégico, pueden representar una categorización de conceptos o variables que se refieren a acciones de individuos u organizaciones. Así, análisis o planificación son categorías conceptuales que implican la realización de una secuencia de actividades que explican como se van formando las estrategias toxicológicas ocupacionales en TOXIMED.

Finalmente, se ha podido comprobar, en la práctica, que los sistemas estratégicos formales no han desaparecido de las organizaciones; por el contrario, se han ido transformando y existe un gran interés por la mejora de los procesos a través de los cuales se generan las ideas para consolidar el pensamiento estratégico. La cuestión no es la capacidad de hacer una reingeniería de los modelos, es la innovación estratégica como clave de la creación de valores y producción de nuevos servicios e investigaciones siendo como bien lo ha expresado Hamel (1998b), el único camino hacia el éxito. Todo el resultado práctico del sistema de gestión en el campo de la toxicología ocupacional, representa una estrategia bien planeada la cual hace referencia y se corresponde con los planes y programas previstos.

\section{Conclusiones}

El modelo dinámico descrito sobre la estrategia planeada a seguir para los servicios científico-técnicos e investigaciones biomédicas en toxicología ocupacional, permitió visualizar, conjuntamente, con las funciones administrativas y directivas las diferentes posibilidades de implementar y coordinar diagnósticos preventivos y de pesquisajes activos con base en asesorías de evaluación de riesgo toxicológico ocupacional, posibilitando determinar y aplicar científicamente nuevas políticas de acción. 


\section{Referencias}

Albert, L.A. (1997). Evaluación de riesgo. En L. Albert (Ed.), Introducción a la toxicología ambiental (pp. 387). Metepec, México: Centro Panamericano de Ecología Humana y Salud. División de Salud y Ambiente.

Castellanos, N.J.E. \& Cruz, P.M. (2014). Una mirada a la evolución histórica de la estrategia organizacional. Revista de Estudios Avanzados de Liderazgo, 1(3), 28-51. Recuperado de http://www. regent.edu/acad/global/publications/ real/vol 1 no3/3...pdf

Cejas, M. (2006). La Formación por competencias: Una visión estratégica en la gestión de personas. Visión General, 4(1), 11-22. Recuperado de http://www.saber.ula.ve/bitstream/123456789/25077/2/articulo2.pdf

Chakravanthy, B.S. \& Doz, Y. (1992). Strategic process research. Strategic Management Journal, 13(special issue), 5-14.

Gastañega, Ma.C. (2012). Salud ocupacional: historia y retos del futuro. Revista Peruana de Medicina Experimental y Salud Pública, 29(2), 177-178. Recuperado de http:// www.scielo.org.pe/pdf/rins/v29n2/ a01v29n2.pdf

Hamel, G. (1998a). Strategy Innovation and the quest for value. Recuperado de http://citeseerx.ist.psu.edu/viewdoc/ download?doi=10.1.1.467.7431\&re$\mathrm{p}=$ rep1 \& type $=\mathrm{pdf}$

Hamel, G. (1998b). Innovación estratégica y creación de valor. Harvard Deusto Business Review, 84, 6-13.

Haro, G.L., Celis, Q.G., López, R.P., Sánchez, R.F.R. \& Juárez, P.C. (2007). Matriz genérica de exposición ocupacional genérica. Revista médica del Instituto Mexicano del Seguro Social, 45(3), 255-263.
Hax, C. \& Majluf, N.S. (1996). The strategic concept and process a pragmatic approach $\left(2^{\text {nd }}\right.$ ed.). New Jersey: Prentice Hall.

Hernández, P.P. (2013). La investigación operativa en salud y su relevancia para lograr los objetivos nacionales e internacionales de desarrollo en Bolivia. Integra Educativa, 6(2), 1-17. Recuperado de http://www.scielo.org.bo/ pdf/rieiii/v6n2/v6n2a11.pdf

Mallar, M.A. (2010). La gestión por procesos: un enfoque de gestión eficiente. Visión de Futuro, 13(1), 1-19. Recuperado de http://www.scielo.org.ar/pdf/ vf/v13n1...pdf

Mintzberg, H. (1994). The rice and fall of strategic planning. New York: Prentice Hall.

Niño, E.J. (2000). Factores, indicadores y marcadores de riesgo en prevención laboral. Mapfre Seguridad, 77, 31-45. Recuperado de https://www.fundacionmapfre.org/documentacion/publico/i18n/catalogo_imagenes/grupo. cmd?path $=1020854$

Organización Mundial de la Salud. (1995). Global Strategy on Occupational Health for All. The Way to Health at Work: Recommendation of the Second Meeting of the WHO Collaborating Centres in Occupational Health. Recuperado de http://www.who.int/occupational_ health/en/oehstrategy.pdf

Organización Mundial de la Salud. (2010). Entornos laborales saludables: fundamentos y modelo de la OMS-Contextualización, prácticas y literatura de apoyo. Recuperado de http://www.who.int/ occupational_health/evelyn_hwp_ spanish.pdf

Quero, L. (2008). Estrategias competitivas: factor clave de desarrollo. Revista Cientifica Electrónica Ciencias Gerenciales NEGOTIUM, 10(4), 36-49. Recuperado en http://www.revistanegotium.org.ve/pdf/10/Art3.pdf 
Rabin, B.A., Brownson, R.C., Haire-Joshu, D., Kreuter, M.W. \& Weaver, N.L. (2008). A glossary for dissemination and implementation research in health. Journal of Public Health Management and Practice, 14(2), 117-23.

Rajagoplan, N. (1997). Strategic orientations, incentive plan adoptions and firm performance: An evidence from Electric Utility Firms. Strategic Management Journal, 10, 761-786.

Rojas, M. (1998). Tópicos de actualidad en toxicología ocupacional. X Congreso Latinoamericano de Toxicología. Palacio de las Convenciones, La Habana, Cuba.

Rubio, D.M., Schoenbaum, E.E., Lee, L.S., Schteingart, D.E., Marantz, P.R., \& et al. (2010). Defining translational research: implications for training. Journal of the Association of American Medical Colleges, 85(3), 470-475.
Sánchez, R. (1995). Strategic flexibility in Product Competition. Strategic Management Journal, 16, 135-159.

Shendel, D. (1992). Introduction to the summer 1992 special issue on strategic process research. Strategic Management Journal, 13, 1-4.

Sussman, S., Valente, T.W, Rohrbach, L.A., Skara, S., \& Pentz, M.A. (2006). Translation in the health professions: converting science into action. Evaluation and the Health Professions, 29(1), 7-32.

Tamayo, R.S. \& Argota, P.G. (2012). Matriz diseñada en toxicología ocupacional. MEDISAN, 16(2), 160-166.

Van de Ven, A.H. (1992). Suggestions for Studying, Strategic Process: A research note. Strategic, process research. Strategic Management Journal, 13, 169188. 
